\title{
Preise und Förderungen der DRG
}

\section{An dieser Stelle möchten wir Ihnen einen Überblick über die Preise und Förderungen der Deutschen Röntgengesellschaft geben. Bitte beachten Sie jeweils die Bewerbungsfristen.}

\section{Wilhelm-Conrad-Röntgen-Preis $\nabla$}

Zum Gedenken an ihren Namensgeber vergibt die Deutsche Röntgengesellschaft seit 1979 jährlich den Wilhelm-ConradRöntgen-Preis. Er ist mit 10.000 Euro dotiert, die Preisverleihung findet während des Deutschen Röntgenkongresses statt.

\section{Auswahlkriterien}

- Der Preis wird verliehen für eine hervorragende wissenschaftliche Arbeit, die dem Fortschritt der Radiologie dient. Eingeschlossen sind die Arbeitsgebiete Radiologische Diagnostik, Strahlentherapie, Strahlenphysik, -biologie und -technik. Die Arbeit muss in deutscher oder englischer Sprache abgefasst sein und sie sollte noch nicht ausgezeichnet worden sein.

- An der eingereichten Arbeit dürfen nicht mehr als drei Autoren beteiligt sein. Der oder die Autoren sollten zudem nicht älter als 40 Jahre alt sein, und sie müssen Mitglieder der Deutschen Röntgengesellschaft oder einer anderen der Europäischen Gesellschaft für Radiologie (ESR) angehörenden nationalen Gesellschaft für Radiologie sein.

\section{Bewerbungsunterlagen und Fristen}

- Die Bewerbungsunterlagen inklusive der wissenschaftlichen Arbeit, einer ausführlichen Zusammenfassung der Arbeit, Lebenslauf mit beruflichem Werdegang, Lichtbild und Schriftenverzeichnis in einfacher schriftlicher Form sowie in elektronischer Form (.doc-Format, .pdf-Format, CD) sind bis zum 30. Oktober 2013 einzureichen an:

\author{
- Deutsche Röntgengesellschaft e.V. \\ Frau Sabine Schlender \\ Ernst-Reuter-Platz 10 \\ 10587 Berlin \\ Mail: schlender@drg.de
}

\section{Walter Friedrich-Preis \\ $\nabla$}

Der Preis würdigt die wissenschaftlichen Leistungen und das prägende Wirken von Prof. Walter Friedrich für die medizinische Radiologie und Strahlentherapie. Das Stiftungsvermögen des Preises entstammt der Gesellschaft für Medizinische Radiologie der ehemaligen DDR und wurde 1992 der DRG übertragen, die das Vermögen 2003 durch eine Zustiftung erhöhte. Der Preis ist mit 2.500 Euro dotiert und wird jährlich während des Deutschen Röntgenkongresses verliehen.

\section{Auswahlkriterien}

- Der Walter-Friedrich-Preis berücksichtigt insbesondere die Gemeinsamkeit von Physik, Technik, Biologie und Medizinischer Radiologie. Er wird verliehen für herausragende wissenschaftliche Arbeiten (incl. Habilarbeiten) auf dem Gesamtgebiet der Radiologie, für beispielgebende Publikationen und Initiativen zur Verbesserung der Lehre auf allen (Teil-)Gebieten der Medizinischen Radiologie und für vorbildliche praktische Ergebnisse in der Medizinischen Radiologie inkl. technischer Entwicklungen.

- Bewerben können sich Einzelpersonen oder Arbeitsgruppen. Der/Die Bewerber sollte/n Mitglied/er der Deutschen Röntgengesellschaft oder einer anderen der ESR angehörenden nationalen Gesellschaft für Radiologie sein und bei Einreichung der Preisarbeiten nicht älter als 40 Jahre sein.

\section{Bewerbungsunterlagen und Fristen}

- Die Bewerbungsunterlagen enthalten die wissenschaftliche Arbeit, eine ausführliche Zusammenfassung der Arbeit, einen Lebenslauf mit beruflichem Werdegang, ein Lichtbild und ein Schriftenverzeichnis. Sie sind in einfacher schriftlicher Form sowie in elektronischer Form (.doc -Format oder PDF, auf CD gespeichert) bis zum 30. Oktober 2013 einzusenden an:

- Deutsche Röntgengesellschaft e.V. Frau Sabine Schlender

Ernst-Reuter-Platz 10

10587 Berlin

Mail: schlender@drg.de

\section{Felix-und-Eugenie-Wachsmann- Preis \\ $\nabla$}

Der Felix-und-Eugenie-Wachsmann-Preis der Akademie für Fort- und Weiterbildung in der Radiologie wurde erstmals 2001 verliehen. Mit ihm werden jährlich Radiologinnen und Radiologen für ihren Einsatz in der radiologischen Weiterbildung ausgezeichnet, die über mehrere Jahre hin erfolgreich am Programm der Akademie mitgearbeitet haben. Mit dem Wachsmann-Preis ehrt die Deutsche Röntgengesellschaft das Andenken des Stifters Prof. Dr. Felix Wachsmann.

Der Preis wird jährlich an fünf Referenten des Akademie-Programms verliehen und ist mit 500 Euro dotiert. Die Gewinner zeichnen sich durch eine langjährige, besonders erfolgreiche Mitarbeit am Akademie-Programm aus. Sie müssen in dem der Verleihung vorangehenden Jahr für mindestens sechs Fortbildungsveranstaltungen von den Teilnehmern sehr gut bewertet worden sein. Die Vergabe erfolgt durch die Auswertung der Fortbildungsevaluationen in der Geschäftsstelle der Deutschen Röntgengesellschaft. 


\section{Werner-Porstmann-Preis zur Förderung der Interventionellen Radiologie \\ $\nabla$}

In Gedenken an Werner Porstmann (1921 - 1982), einen Pionier der kardiovaskulären Interventionen, schreiben die Deutsche Röntgengesellschaft und die Deutsche Gesellschaft für Interventionelle Radiologie und minimalinvasive Therapie (DeGIR) den Werner-Porstmann-Preis zur Förderung der Interventionellen Radiologie aus. Der Preis ist mit 7.500 Euro dotiert; das Preisgeld wird von der Firma Boston Scientific Medizintechnik GmbH gespendet. Der Preis wird jährlich auf dem Deutschen Röntgenkongress durch den Vorsitzenden der DeGIR überreicht.

\section{Auswahlkriterien}

- Der Preis wird jährlich verliehen für die beste wissenschaftliche Arbeit auf dem Gebiet der Interventionellen Radiologie, die in den zwei der Verleihung vorausgehenden Jahren veröffentlicht bzw. zur Veröffentlichung angenommen wurde. Der einreichende Autor sollte zum Bewerbungszeitpunkt nicht älter als 35 Jahre sein, und er muss Mitglied der DRG und der DeGIR sein. Den Hauptteil der zugrundeliegenden Studien sollte der Bewerber hauptsächlich selbständig durchgeführt haben. Es darf nur eine Publikation eingereicht werden.

\section{Bewerbungsunterlagen und Fristen}

- Die Bewerbungsunterlagen enthalten die wissenschaftliche Arbeit, einen kurzen Lebenslauf sowie ein Schriftenverzeichnis der letzten zwei Jahre, an denen der einreichende Autor als Erstoder Letztautor beteiligt war. Bei Veröffentlichungen mit mehreren Autoren ist der Bewerbung eine Bestätigung des Vorgesetzten beizufügen, die ausweist, dass der einreichende Autor den entscheidenden Anteil an der Veröffentlichung trägt.

- Die Bewerbungsunterlagen sind in einfacher schriftlicher Form sowie in elektronischer Form (.doc -Format oder PDF, auf CD gespeichert) bis zum 15. Februar 2014 einzusenden an:

- Deutsche Röntgengesellschaft e.V. Frau Sabine Schlender

Ernst-Reuter-Platz 10

10587 Berlin

Mail: schlender@drg.de

\section{Alfred-Breit-Preis $\nabla$}

Der Preis erinnert an Prof. Alfred Breit, einen der Wegbereiter der Strahlentherapie. Der Preis wird seit 2012 jährlich vergeben und ist mit 20.000 Euro dotiert. Die Verleihung findet während des Deutschen Röntgenkongresses statt.

\section{Auswahlkriterien}

- Die Kriterien für den Alfred-Breit-Preis sind testamentarisch festgelegt. Der Preis wird für Arbeiten und Entwicklungen vergeben, die aus dem Bereich der radiologischen Forschung kommen und erkennbar zu Fortschritten in der Krebstherapie beitragen können. Als Grundlage zur Entscheidung für die Preisvergabe können Publikationen oder Patente dienen, aber auch Verfahren, die sich in der Klinik etabliert haben, ohne dass sie publiziert worden sind.

- Der Preis kann an einzelne Wissenschaftler, aber auch an Arbeitsgruppen oder Institutionen vergeben werden. Kandidaten können auch von Dritten vorgeschlagen werden; Voraussetzung hierfür ist ein abgeschlossenes wissenschaftliches Studium bzw. eine entsprechende abgeschlossene Ausbildung der/des Kandidatin/Kandidaten.

\section{Bewerbungsunterlagen und Fristen}

- Eine kurze Zusammenfassung der Arbeit / des Patents / des klinischen Verfahrens (max. 2 DIN A4-Seiten)

- Eine Kopie der Publikation(en)/ des Patents bzw. eine ausführliche Beschreibung des für den Preis vorgeschlagenen Verfahrens

- einen Lebenslauf und des Erstautors / der Erstautorin bzw. des Leiters / der Leiterin der Arbeitsgruppe bzw. des Patentinhaber/der Patentinhaberin

- ein Publikationsverzeichnis derselben Person

- bei Bewerbungen von Einzelpersonen: ein Lichtbild

- Die Bewerbungsunterlagen sind bis zum 31. Januar in einfacher schriftlicher Form sowie auch in elektronischer Form (doc-Format oder PDF, auf CD gespeichert) einzureichen an:

- Deutsche Röntgengesellschaft e.V. Frau Sabine Schlender

Ernst-Reuter-Platz 10

10587 Berlin

Mail: schlender@drg.de
Promotionspreis der Deutschen Röntgengesellschaft

$\nabla$

Der Promotionspreis der Deutschen Röntgengesellschaft wurde erstmals zum Jahr 2012 ausgeschrieben, um wissenschaftlich engagierte Studenten und Mediziner zu motivieren und zu fördern. Pro Jahr werden bis zu drei Promotionsarbeiten ausgezeichnet. Im Fall einer Auszeichnung der eingereichten Arbeit wird diese als Originalarbeit in der Fachzeitschrift RöFo veröffentlicht. Der Preis ist mit 1.000 Euro und der kostenlosen Teilnahme am kommenden Deutschen Röntgenkongress dotiert.

\section{Auswahlkriterien}

- Teilnehmen können Bewerber, die im vorhergehenden Jahr auf dem Gebiet der Radiologie und Medizinphysik promoviert haben. Die eingereichte Arbeit resultiert aus der Promotion (bzw. aus Teilen der Promotion) und ist in Form einer wissenschaftlichen Originalarbeit nach den Richtlinien für Autoren der RöFo abgefasst. Von einer Fachzeitschrift bereits veröffentlichte oder zur Publikation angenommene Arbeiten, die aus der Promotion des Bewerbers resultieren, können ebenfalls für den Preis eingereicht werden. Sie werden, falls sie für den Preis ausgewählt werden, nach ihrer Veröffentlichung in der RöFo referiert.

\section{Bewerbungsunterlagen und Fristen}

- Die Bewerbungsunterlagen enthalten die wissenschaftliche Arbeit, einen kurzen Lebenslauf und ein Lichtbild. Sie sind in einfacher schriftlicher Form sowie in elektronischer Form (.doc Format oder PDF, auf CD gespeichert) bis zum 30. Oktober 2013 einzusenden an:

- Deutsche Röntgengesellschaft e.V. Frau Sabine Schlender Ernst-Reuter-Platz 10 10587 Berlin Mail: schlender@drg.de

\section{Young Investigator Award \\ $\nabla$}

Auf Initiative der AG Methodik und Forschung werden seit dem Jahr 2009 alljährlich anlässlich des Deutschen Röntgenkongresses mit dem Young Investigator Award herausragende Arbeiten und Kongressbeiträge junger Wissenschaftler ge- 
ehrt. Mit dem YIA sollen die Leistungen des wissenschaftlichen Nachwuchses in der Bildgebung herausgehoben und gefördert werden. Die Tatsache, dass von den bisherigen Preisträgern mehr als die Hälfte Naturwissenschaftler waren, mag als Zeichen dafür gesehen werden, dass der Award vor allem sehr innovative Forscher anlockt. Der Preis ist mit 500 Euro dotiert.

\section{Auswahlkriterien}

- Für den YIA können sich Wissenschaftler unter 35 Jahren mit ihren Arbeiten im Rahmen der regulären Abstracteinreichung bewerben. Die für den YIA eingereichten Abstracts sollen in Form und Struktur den Kriterien für reguläre Beiträge zum Deutschen Röntgenkongress folgen. Die Beiträge durchlaufen ein intensiveres und strengeres Review als die üblichen Kongressbeiträge; nur solche Abstracts, die von beiden Gutachtern unabhängig als exzellent bewertet werden, werden zum Vortrag auf dem Kongress eingeladen. In den dezidierten YIA Sessions stellen die jungen Wissenschaftler ihre Arbeit einer Jury renommierter deutscher Wissenschaftler vor, nach deren Votum der Preis verliehen wird.

\section{Bewerbung}

- Die Bewerbung erfolgt im Rahmen der regulären Abstracteinreichung für den Deutschen Röntgenkongress.

\section{Reisestipendien der Deutschen Röntgengesellschaft}

Zur Förderung des wissenschaftlichen Nachwuchses in der Radiologie lobt die Deutsche Röntgengesellschaft für Autoren von Vorträgen und Abstracts, die für europäische Radiolgiekongresse in Deutschland angenommen wurden, je 10 Reisestipendien zu 500 Euro aus den Erträgen der Wilhelm Conrad Röntgen-Stiftung aus. Über die Aufnahme eines europäischen Radiologiekongresses in das Stipendienprogramm entscheidet der Vorstand der Deutschen Röntgengesellschaft.

\section{Auswahlkriterien}

- Der Kongress findet in Deutschland unter der Leitung eines Mitglieds der Deutschen Röntgengesellschaft statt. Die/der Bewerber/in sollte Mitglied der Deutschen Röntgengesellschaft und zum Zeitpunkt des Kongresses möglichst nicht älter als 35 Jahre alt sein. Sie/er sollte Erstautor/in eines Beitrags (Vortrag oder Poster) sein, der in das wissenschaftliche Programm des Kongresses aufgenommen wurde.

Bewerbungsunterlagen und Fristen

- Das Antragsformular können Sie auf unserer Homepage www.drg.de herunterladen.

- Der Antrag muss Ort, Zeit und Thema des Kongresses enthalten sowie einen kurzen Lebenslauf und die Bestätigung der Annahme eines Vortrages/Posters. Der Antrag muss spätestens 6 Wochen vor dem geplanten Kongressbesuch in einfacher schriftlicher Form sowie in elektronischer Form (.doc-Format, . pdf-Format, CD) an die Geschäftsstelle der DRG gerichtet werden:

- Deutsche Röntgengesellschaft e.V. Frau Sabine Schlender Ernst-Reuter-Platz 10 10587 Berlin Mail: schlender@drg.de

\section{Reisekostenzuschüsse der Rajews- ky-Stiftung}

Die Rajewsky-Stiftung gewährt eine begrenzte Anzahl von Reisekostenzuschüssen zum Besuch der internationalen Kongresse für Radiologie. Die Zuschüsse werden aus den Zinserträgen der Stiftung gewährt.

\section{Auswahlkriterien}

- Bewerben können sich junge, wissenschaftlich engagierte, deutsche Radiologen/Innen, die nicht älter als 40 Jahre alt und Mitglied/er der DRG sind. Der/die Antragsteller/in sollte/n auf den Kongressen, für die Zuschüsse beantragt werden, wissenschaftliche Vorträge halten.

\section{Bewerbungsunterlagen und Fristen}

- Der Antrag muss Ort, Zeit und Thema des Kongresses enthalten sowie die Bestätigung der Annahme eines Vortrages. Der Antrag sollte spätestens 10 Wochen vor dem geplanten Kongressbesuch in einfacher schriftlicher Form sowie in elektronischer Form (.docFormat, .pdf-Format, CD) bei der Geschäftsstelle der DRG eingereicht werden: 


\author{
- Deutsche Röntgengesellschaft e.V. \\ Frau Sabine Schlender \\ Ernst-Reuter-Platz 10 \\ 10587 Berlin \\ Mail: schlender@drg.de
}

\section{Reisestipendium der Rolf-W.-Gün- ther-Stiftung zum ECR \\ $\nabla$}

Die Rolf W. Günther Stiftung schreibt für Autoren von Vorträgen zum Europäischen Röntgenkongress in Wien (ECR) fünf Reisestipendien über 500 Euro aus. Ziel der Stiftung ist die Förderung der Wissenschaft und Forschung auf dem Gebiet der Radiologischen Wissenschaften insbesondere der diagnostischen und interventionellen Radiologie sowie der nationalen und internationalen Zusammenarbeit auf diesem Gebiet.

\section{Auswahlkriterien}

- Der Antragsteller / die Antragstellerin sollte Mitglied der Deutschen Röntgengesellschaft sein, zum Zeitpunkt des Kongresses möglichst nicht älter als 35 Jahre alt und Erstautor/in eines Vortrages sein, der in das wissenschaftliche Programm des ECR aufgenommen wurde.

\section{Bewerbungsunterlagen und Fristen}

- Das Antragsformular können Sie auf unserer Homepage www.drg.de herunterladen.

- Der Antrag muss Ort, Zeit und Thema des Kongresses enthalten sowie die Bestätigung der Annahme eines Vortrages/Posters. Der Antrag muss spätestens 6 Wochen vor dem geplanten Kongressbesuch in einfacher schriftli- cher Form sowie in elektronischer Form (.doc-Format, .pdf-Format, CD) bei der Geschäftsstelle der DRG eingereicht werden:

- Deutsche Röntgengesellschaft e.V. Frau Sabine Schlender Ernst-Reuter-Platz 10 10587 Berlin Mail: schlender@drg.de

\section{Kongresspatenschaft „die hellsten Köpfe für die Radiologie“"}

Die DRG unterstützt seit 2009 individuelle Förderer der Radiologie, die Medizin- studenten zum Deutschen Röntgenkongress einladen und sie so für die Radiologie begeistern möchten, mit einem umfassend organisierten Patenschaftsprogramm.

\section{Hellste-Koepfe.}

Patenschaftsprogramm.

- Die hellsten Köpfe für die Radiologie! Auf www.hellste-koepfe.de erfahren Sie, was das Kongresspaket enthält und wie die Anmeldung funktioniert. 